\title{
HST-NICMOS observations of extensive triggered star formation within the 30 Doradus Nebula
}

\author{
Nolan R. Walborn and Rodolfo H. Barbá \\ Space Telescope Science Institute, Baltimore, MD 21218, USA
}

\begin{abstract}
The extensive 'second generation' of star formation within the 30 Doradus Nebula, evidently triggered by the R 136 central cluster around its periphery, has been imaged with HST-NICMOS. Many new infrared sources and structures are found in these images.
\end{abstract}

A groundbased, blue-violet spectral classification study of the 30 Doradus stellar content by Walborn \& Blades (1997) revealed five spatially and/or temporally distinct components. One of them is the central ionizing cluster including $\mathrm{R} 136$, corresponding to the Carina phase of $\mathrm{OB}$ cluster evolution, with an age of 23 Myr. HST-FOS spectroscopy by Massey \& Hunter (1998) has shown that twothirds of the 60 brightest stars in and around R 136 are of spectral type O3, the richest concentration known of these most massive young stars. An even younger, Orion-phase generation of age $<1 \mathrm{Myr}$, in or near the bright nebular filaments west and northeast of $\mathrm{R} 136$, contains heavily embedded early-O dwarfs and IR sources, and its formation has likely been triggered by the central cluster.

Recent optical, IR, and radio evidence for current star formation in 30 Dor is reviewed by Rubio et al. (1998) and Walborn et al. (1999). The latter paper presents first results from an HST-NICMOS survey, which because of its 0 ' 1 resolution provides new insights. Many new IR sources, including multiple systems, clusters, and nebular structures, are found in these NICMOS images. Knots 1-3 of Walborn \& Blades (1997), early O-type stars embedded in dense nebular knots, are all found to be compact multiple systems.

Knot 1, northeast of $R 136$, resides at the top of a massive dust pillar oriented directly toward R 136, whose summit has just been removed, exposing the newborn stellar system. Two of the brightest IR sources in 30 Dor, with only very faint counterparts in $I$, are found in an adjacent pillar with the same orientation just northeast of Knot 1 . Knot 2, also northeast of $R 136$, is associated with detached, nonstellar IR sources aligned on either side of the stellar system, which are interpreted as impact points of a highly collimated, possibly rotating, parsec-scale bipolar jet on the surrounding dark clouds; there is also an $\mathrm{H}_{2} \mathrm{O}$ maser in this field. Another very bright IR source with a very faint optical counterpart is found near Knot 3 , within the head of a pillar west of and also oriented toward R 136. There are several parsec-scale jet structures near Knot 3 (see Figure 1), some of which may be associated with fainter IR stars nearby.

The outflows from young massive stars in 30 Dor are the first extragalactic examples of the phenomenon. These results establish the 30 Dor nebula as a prime region in which to investigate the formation and very early evolution of massive stars and multiple systems; several sequential stages and their interstellar interactions are directly observable in the optical and IR. The theme of 

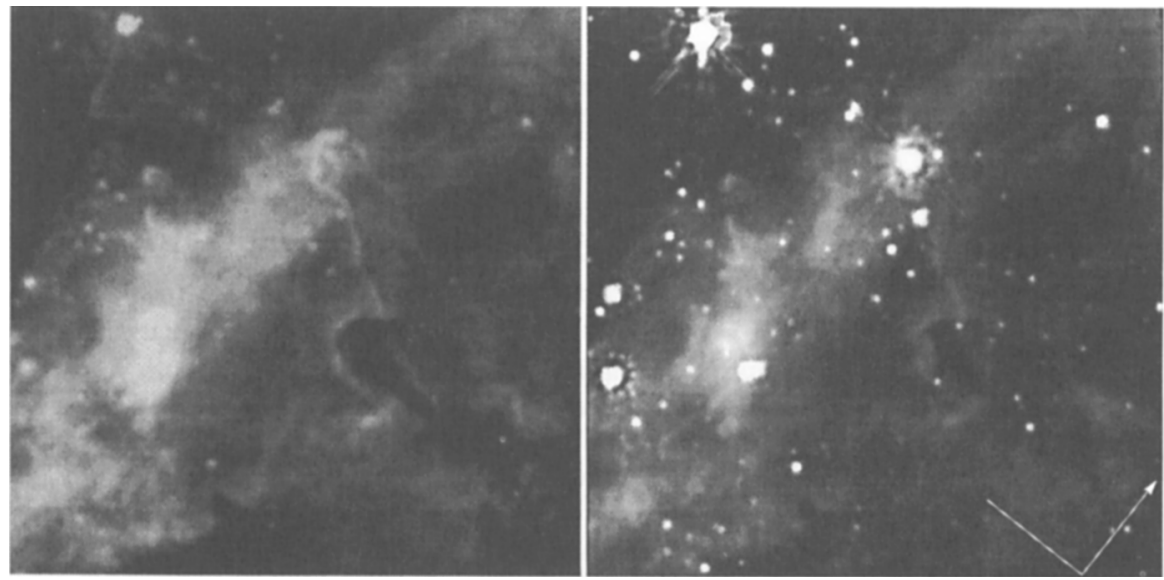

Figure 1. The field of Knot 3 in 30 Dor (Walborn \& Blades 1997). Left: a $H S T$-WFPC2 $\mathrm{H} \alpha$, [S II] composite. Right: a HST-NICMOS JHK composite with the same orientation and scale; the scale bars indicate $\mathrm{N}, \mathrm{E}$ and are $4^{\prime \prime}=1 \mathrm{pc}$ in length.

triggered formation within the heads of extensive dust pillars oriented toward $\mathrm{R} 136$ is strong. In addition, these results provide further insights into the global structure and evolution of 30 Dor, which are significant in view of its status as the best resolved extragalactic starburst. As discussed by Scowen et al. (1998), the most intense nebular emission arises from filamentary interfaces between the central cavity and surrounding molecular clouds. The IR results show that these interfaces are in fact star-formation fronts moving into the dark clouds. 30 Dor is a two-stage starburst, in which a second generation of massive stars is being triggered around the periphery of the initial, massive central cluster. It will evolve into a giant shell HII region like N 1 in the LMC (Walborn \& Parker 1992) and NGC 604 in M 33. There are clear implications for the interpretation of more distant starbursts. E.g., the HST-wFPC2 image of the galactic-scale starburst in NGC 4214 (Mas-Hesse \& Maíz-Apellániz, these Proceedings) reveals that it consists of a collection of 30 Dor- and N 11-like components. These individual regions would subtend less than $1^{\prime \prime}$ each at the Virgo Cluster.

Acknowledgments. The NICMOS program is being conducted in collaboration with W. Brandner, E. Grebel, R. Probst, and M. Rubio. Support for this work was provided by NASA through grants numbers GO-7819.01-96A and AR-7545.01-96A from STScI, which is operated by AURA, Inc., under NASA contract NAS 5-26555.

\section{References}

Rubio, M., Barbá, R., Walborn, N.R., Probst, R.G., et al. 1998, AJ 116, 1708 Massey, P., Hunter, D.A. 1998, ApJ 493, 180

Scowen, P.A., Hester, J.J., Sankrit, R., Gallagher, J.S., et al. 1998, AJ 116, 163

Walborn, N.R, Barbá, R., Brandner, W., Rubio, M., et al. 1999, AJ 117, 255

Walborn, N.R., Blades, J.C. 1997, ApJS 112, 457

Walborn, N.R., Parker, J.Wm. 1992, ApJ 399, L87 